\title{
A convenient and adjustable surface-modified complex containing poly-L-glutamic acid conjugates as a vector for gene delivery
}

\author{
Ye Sun' \\ Yuzhao Tang' \\ Maoquan $\mathrm{Chu}^{2}$ \\ Shuxian Song' \\ Yanfei Xin ${ }^{3}$ \\ 'School of Life Science and \\ Technology, Shanghai Jiaotong \\ University, Shanghai, China; ${ }^{2}$ Institute \\ of Life Science and Technology, Tongji \\ University, Shanghai, China; ${ }^{3}$ School \\ of Pharmacy, Shanghai Jiaotong \\ University, Shanghai, China
}

\begin{abstract}
In order to quantify the amount of ligands or poly(ethylene glycol) (PEG) on each vector, here we developed a system in which poly-L-glutamic acid (PLG) was used as surface modification loading backbone, to which one PEG (MW 5000, 10000, 20000) or epidermal growth factor (EGF) was linked. The PLG conjugates can electro-statically adsorb upon DNA/ polycation complex with positive charge, and, the amount of EGF or PEG on the surface of complexes could be varied. We have made a series of complexes containing the various PLG conjugates and examined their physicochemical properties, and made a comparison of properties and transfection efficiency between these complexes. EGF- and PEG-modified complexes showed 10-25-folds higher cell transfection efficiency than unmodified complexes in medium with or without serum.
\end{abstract}

Keywords: gene delivery, poly(ethylene glycol), poly-L-glutamic acid, epidermal growing factor, poly-L-lysine

\section{Introduction}

In recent years, nonviral gene delivery system was widely used in gene delivery due to no or little risk of immune response and large-scale manufacturing compared with viral gene carriers (Merdan et al 2002; Ogris and Wagner 2002).

Sukhorukov and colleagues (1998) showed that the development of nanoparticles by stepwise layer-by-layer deposition of alternatively charged polyelectrolyte onto polystyrene and melamine formaldehyde latex particles that are able to encapsulate different pharmacological agents, proteins, and so on. Based on their studies, Zaitsev and colleagues (2004) made some compact particles with negative charge and low aggregation capacity under physiological conditions for nonviral gene delivery systems by depositing polyanion (PVS or transfer RNA) to DNA/polyethylenimine (PEI) complex. This approach was firstly used for DNA encapsulation with a DNA/ poly-L-lysine (PL L) core by Trubetskoy and colleagues (1999), in which PLL and succinylated PLL as polycation and polyanion, respectively, was used to deposit alternating layer of polyanion and polycation on the surface of positive DNA/PLL complex. These complexes with layer of alternating layer of polyanion and polycation was physicochemically characterized. Transfection activity of these complexes was shown by these authors later (Trubetskoy et al 2003). Maruyama and colleagues (2004) used PEG derivative containing carboxylic acid and lactose side chain (Lac-PEG-C) to deposit on the surface of DNA/protamine complex with positive charges, and then obtained 56-fold higher transfection efficiency with DNA/protamine/Lac-PEG-C at weight ratio 1:1.5:8 than that without Lac-PEG-C (Maruyama et al 2004). Other researchers also made use of DNA/polycation complexes modified with PEG derivatives having carboxylic acid and/or sugar and received certain 
effects (Koyama et al 2002, 2003). These DNA/polycation complexes modified with PEG derivatives showed a strong resistance against the albumin-induced aggregation and enhanced transfection efficiency compared to DNA/polycation complexes without modification. But in above investigations the amount of carboxylic acid and ligand in the line of PEG derivative was discrete, so didn't determine the amount of these molecules in PEG derivative. Finsinger and colleagues (2000) linked peptide with negative charge such as INF7 or YE5C to glutamic acid on PEG-glutamice acid copolymer, then modified it on the surface of DNA complexes formed with PEI, PLL, or liposome, which obtained 2-20-folds higher efficiency than control. However there is a lot of interferon-7 (INF7) or YE5C in each PEG-glutamic acid copolymer in this approach. Although these peptide made PEG to closely adsorb on the surface of complex, these discrete peptide in each PEG-glutamic acid copolymer maybe cause some complexes to aggregate to large complexes even precipitation.

In our study, we showed a nonviral gene delivery system composed of PLL-, DNA-, and PLG-conjugated PEG or EGF. Here we used PLG as a bridge between DNA/PLL complex and PEG or EGF. There is one PEG or EGF conjugated to each PLG through sole end amino group of PLG. The advantage of this system is that each complex with various amounts of PEG and/or EGF can be produced easily without making a new synthesis, and PEG or EGF can maximally stand at the surface of complexes. Then we can adjust the amount of PEG or EGF in this system as long as controlling the amount of PLG. So we were able to conveniently investigate the relation between the amounts of PEG and/or EGF in complexes and physicochemical property or cell transfection efficiency of complexes.

\section{Materials and methods}

PLL (MW $=28,500$ ) was purchased from Sigma Chemical (St Louis, MO, USA) and used without further purification. Recombinant human EGF (rhEGF) was purchased from Shanghai Bioengineering center (Shanghai, China). PLG (20 glutamic acid, purity: $\geq 95 \%$ ) was synthesized by Shanghai Sangon Biological Engineering Technology and Service Co., Ltd (Shanghai, China). Succinimidyl 4-[N-maleimidomethyl] cyclohexane-1-carboxylate (SMCC), N-succinimidyl 3-[2-pyridyldithio]-propionamido (SPDP) and slide-A-lyzer dialysis unit (3500, 10000 MWCO) were purchased from Pierce Biotechnology. Inc. (Rockford, IL, USA). sc-mPEG (MW = 5000, 10000, 20000, D $=1.12$, sc cont: $>90 \%$ ) were synthesized by Xinfeng Biotechnology Co., Ltd (Shanghai, China). Fusedsilica capillary ( $75 \mu \mathrm{m}$ ID) was purchased from Yongnian Optical Fiber Factory (Hebei, China). All other reagents were of analytical grade.

\section{Cell culture}

Human hepatoma cell line SMMC-7721 (a gift from Shanghai Cancer Institute, Shanghai Jiaotong University, China) was cultured in DMEM supplemented with $10 \%$ fetal bovine serum (FBS) at $37{ }^{\circ} \mathrm{C}$ in $5 \% \mathrm{CO}_{2}$ atmosphere.

\section{Synthesis of PEG-g-PLG copolymers}

PEG-g-PLG was synthesized by coupling the amino group of PLG with various sc-mPEG as previously described (Guo et al 2004) with small modification (Scheme 1). Briefly, PLG and sc-mPEG (at molar ratio of 6:1) were dissolved in $0.05 \mathrm{M}$ phosphate buffer (pH 7.4) and DMSO respectively. sc-mPEG was added to the PLG solution quickly and the solution was stirred under $\mathrm{N}_{2}$ atmosphere at room temperature for 8 hours, then dialyzed against ultra-pure water in dialysis unit (3500 MWCO) twice for $3 \mathrm{~h}$ each.

\section{Conjugation of PLG and EGF}

Conjugation of PLG and EGF was referred to the method (Wagner et al 1990) with small modification (Scheme 2). Briefly, (1) $0.113 \mathrm{mg}$ SPDP in DMSO was added to $1.5 \mathrm{mg}$ EGF (the molar ratio of SPDP to EGF is 1.5:1) in phosphate buffers ( $\mathrm{pH}$ 7.5) and quickly mixed. After reaction for 60 min at room temperature, the product was dialyzed against deionic water twice for $3 \mathrm{hr}$ each by using dialysis unit (3500 MWCO), and then lyophilized for next procedure. (2) Lyophilized EGF-SPDP was redissolved in acetate acid buffer ( $\mathrm{pH} 4.5$ ), in which $1.84 \mathrm{mg} \mathrm{1,} \mathrm{4-Dithiothreitol}$ (DTT) (the molar ratio of DTT to SPDP is 17:1) was

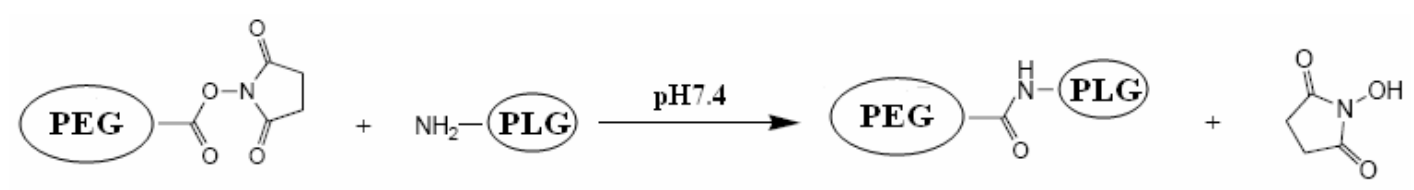

Scheme I The schematic picture of PLG grafted with PEG. 
added to activate the dithiol of SPDP on EGF-SPDP into sulphydryl group. After reaction for 30 min under $\mathrm{N}_{2}$ at room temperature, the product was dialyzed against deionic water twice for $3 \mathrm{~h}$ each by using dialysis unit (3500 MWCO), and then lyophilized for next procedure. (3) $0.8 \mathrm{mg} \mathrm{SMCC}$ in DMSO was added to $6.3 \mathrm{mg}$ PLG (the molar ratio of SMCC to PLG is $0.8: 1$ ) in phosphate buffer ( $\mathrm{pH}$ 7.4) and quickly mixed. After reacting for $60 \mathrm{~min}$ at room temperature, the product was ready for next procedure. (4) Lyophilized EGF-SPDP with sulphydryl group was dissolved in phosphate buffer ( $\mathrm{pH}$ 7.4) and then added into PLG-SMCC solution under stirring. The mixture was incubated for $20 \mathrm{~h}$ at 10 degree celsius. After reaction, the product was dialyzed against deionic water twice for $3 \mathrm{~h}$ each by using dialysis unit (3500 MWCO) and then lyophilized.

\section{Preparation of coated capillary}

The coated capillary in this study was prepared by our lab as previous described (Zhang et al 2004). Firstly capillary was washed by $0.1 \mathrm{M} \mathrm{HCl}, 0.1 \mathrm{M} \mathrm{NaOH}$, and, water respectively. Then the inside wall of capillary was silanylated by 3-(trimethoxysilyl) propyl methacrylate. Finally the inside wall of capillary was coated by N, N-dimethylacrylamide. The coated capillary can be used after it has aged at room temperature overnight.
Characterization of PEG-g-PLG and EGF-g-PLG by capillary electrophoresis (CE)

A P/ACE ${ }^{\mathrm{TM}}$ MDQ capillary electrophoresis system (Beckman, Fullerton, CA, USA) was equipped with the coated capillary ( $75 \mu \mathrm{m}$ inner diameter). Samples were injected by pressure and the on column UV detection was set at $214 \mathrm{~nm}$. The temperature of the capillary cartridge was maintained at $25 \pm 0.1^{\circ} \mathrm{C}$

\section{Preparation of DNA/PLL/PLG conjugate complex}

PLL solution with concentration of $1 \mathrm{mg} / \mathrm{ml}$ (the total charge ratio of amino group in PLL to phosphate in DNA and carboxylic acid in PLG is 1:5, 1:3, 1:1, 3:1, or 5:1) was quickly mixed with $1 \mu \mathrm{g}$ of pGL3 plasmid DNA with concentration of $1 \mathrm{mg} / \mathrm{ml}$ in HEPES buffer ( $\mathrm{pH} 7.3,10 \mathrm{mM}$ ) respectively and the mixture was incubated for $15 \mathrm{~min}$ at room temperature. Then PLG (or PEG-g-PLG, EGF-g-PLG) with concentration of $0.5 \mathrm{mg} / \mathrm{ml}$ (the charge ratio of phosphate in DNA to carboxylic acid in PLG is $1: 5,1: 3,1: 1,3: 1$, or 5:1) was incubated with DNA/PLL complex for $15 \mathrm{~min}$ at room temperature, respectively.

\section{Size and zeta potential of DNA complex}

The size and zeta potential of DNA and polymers complexes were measured by Photon Correlation Spectroscopy (PCS)

reaction 1:

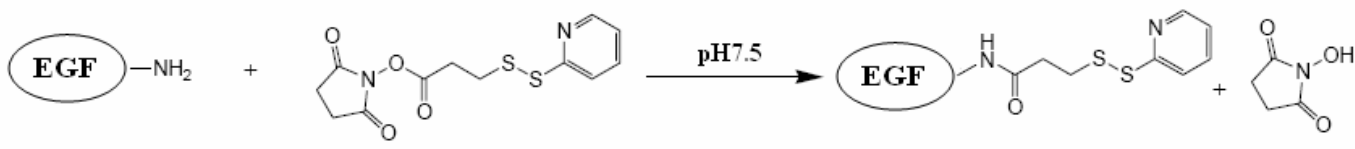

reaction 2:
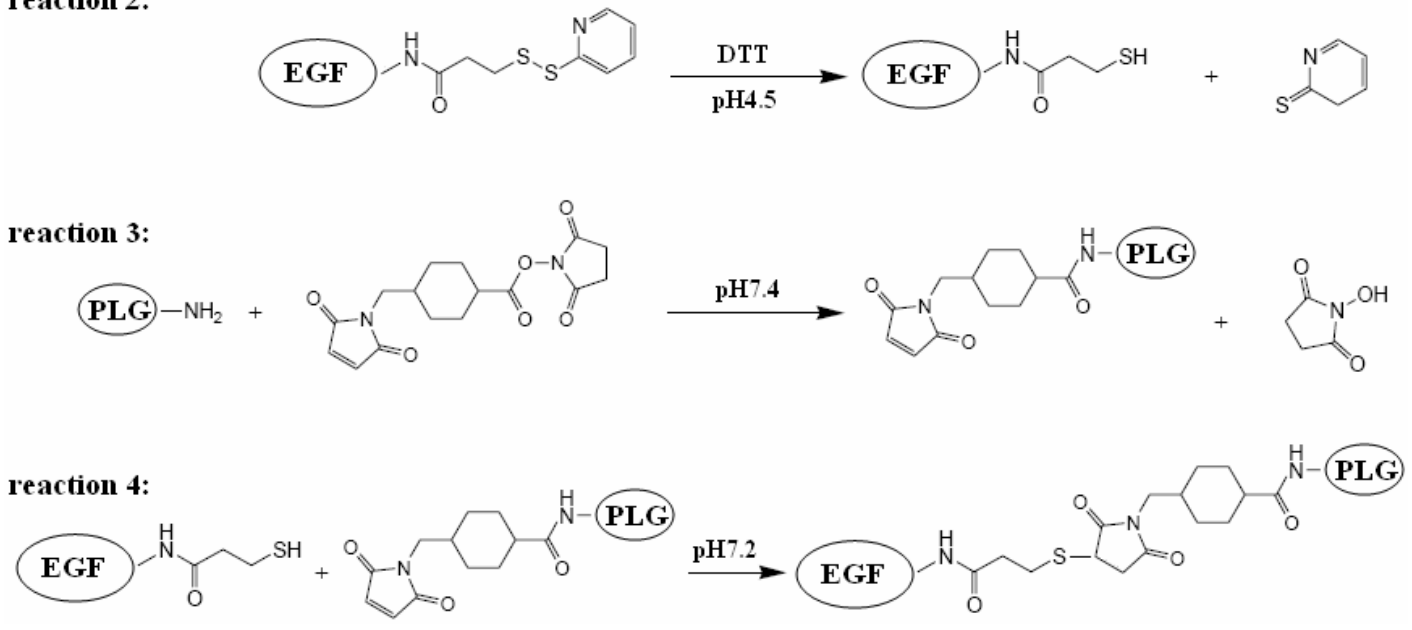

Scheme 2 The schematic picture of PLG grafted with EGF. 
(Malvern Instruments Ltd. Worcestershire, UK) equipped with a He-Ne laser of $633 \mathrm{~nm}$. DNA and polymers complexes with different composition was injected into detector and measured at $25^{\circ} \mathrm{C}$ with the constant angle of $90^{\circ}$.

\section{Nuclease resistance of DNA complexes}

The ability of polymers to protect DNA from enzymatic degradation was evaluated by nuclease resistance assay as previous described (Lee et al 2001). Briefly, $20 \mu 1$ DNA complexes ( $1 \mu \mathrm{g}$ DNA) were mixed with DNase I ( 1 unit/1 $\mu \mathrm{g}$ DNA) and the mixture was incubated for $20 \mathrm{~min}$ at $37^{\circ} \mathrm{C}$. Then the DNase I digestion was stopped by equal volume of stop solution ( $0.8 \mathrm{M} \mathrm{NaCl}, 50 \mathrm{mM}$ EDTA) and $2 \mu \mathrm{l}$ of $10 \%$ SDS was added to the mixture to disassociate DNA from the complexes at $65^{\circ} \mathrm{C}$ overnight. DNA was extracted from the mixture by the phenol/chloroform solution and precipitated in $75 \%$ ethanol, then applied to $0.8 \%$ agarose gel electrophoresis.

\section{Cell transfection assay}

In vitro transfection efficiency of DNA complexes was tested in SMMC-7721 cells. Complexes containing $1 \mu \mathrm{g}$ DNA were added into each well in the medium supplemented with or without $10 \%$ FBS. After incubating for $6 \mathrm{hr}$, medium with complexes was removed and substituted by $0.5 \mathrm{ml}$ medium with $10 \%$ FBS. Cells were incubated for another $42 \mathrm{~h}$. At the end of incubation, medium was removed and $200 \mu \mathrm{l}$ of lysis buffer was added into each well to disassociate cells from plate. The lysed cells were collected and spun down. $50 \mu \mathrm{l}$ of supernatant were mixed with $50 \mu$ l of luciferase reagent (Promega Corp, Wisconsin, USA), the luminescence value were detected by the TD-20/20 luminometer (Turnerbiosystems Inc, California, USA).

\section{Results and discussions Characterization of PEG-g-PLG and EGF-g-PLG}

When PLG was linked to PEG of various lengths, peak position of PEG-g-PLG was obviously different when running in CE (Figure 1A). The longer the length of PEG, the shorter the peak time of PEG-g-PLG was. The peaks of PLG, EGFg-PLG and EGF were approximately appeared at 5th min, 22nd min, and 29th min, respectively (Figure 1B).

\section{Size and zeta potential of DNA complex}

The size of complex gradually increased from about $58 \mathrm{~nm}$, which was the size of DNA/PLL, when PEG-g-PLG was added at PLG to DNA charge ratio below 1 (Figure 2A). At the PLG to DNA charge ratio of 1 , the largest size of complex containing PEG $_{5000}$-g-PLG, PEG $_{10000}$-g-PLG or PEG $_{20000}$-g-PLG was about 78, 83, and $88 \mathrm{~nm}$, respectively. Meanwhile the polydispersity index of these complexes, which was at the ranges of 0.19 to 0.29 , didn't obviously change (Figure 2C). So it was believed that the addition of PEG-g-PLG to DNA/PLL didn't lead to aggregate. In comparison, aggregation was observed by adding PLG alone in DNA/PLL complex (data not shown). The size of complex was correlated with the molecular weight of PEG-g-PLG, ie, the size of complex containing PEG ${ }_{20000^{-}}$ g-PLG is larger than the size of complex containing $\mathrm{PEG}_{10000^{-}}$ g-PLG, which is larger than the size of complex containing $\mathrm{PEG}_{5000}$-g-PLG (Figure 2B). At PLG to DNA ratio below 1, the size of complexes containing $\mathrm{PEG}_{20000}$-g-PLG, $\mathrm{PEG}_{10000^{-}}$ g-PLG or PEG $_{5000}$-g-PLG increased to $88 \mathrm{~nm}, 77 \mathrm{~nm}$, and 71 $\mathrm{nm}$, respectively. At the same time the polydispersity index of complexes at about 0.22 didn't obviously change (Figure 2D). It was believed that the addition of PEG-g-PLG did not lead to aggregation of complexes. When PLG to DNA charge ratio in complex was more than 1 , further increase this ratio resulted in decrease of the complex size (Figure 2B). Meanwhile the polydispersity index of complexes gradually increased at PLG to DNA charge ratio above 1 (Figure 2D). This might be due to the binding of some PEG-g-PLG and excessive free PLL leading to formed smaller complex, so that the average size of complexes decreased and the polydispersity index of complexes increased at PLG to DNA charge ratio above 1.

The $\delta$-potential of complex containing PLG gradually decreased with increasing the amount of PLG in complex until PLG to DNA charge ratio of 2 , at which ratio the $\delta$-potential was about $-30 \mathrm{mV}$ (Figure $3 \mathrm{~A}$ ). It was believed that PLG was bind to DNA/PLL complex. This result was in agreement with the previous observations by Trubetskoy and colleagues (1999) and Finsinger and colleagues (2000), in which polyelectrolytes with negative charge could be attached to the surface of condensed DNA particles and obviously decreased the zeta-potential of complexes. Trubetskoy and colleagues (1999) have observed that at the charge stoichiometry of DNA/PLL/SPLL close to 1:1:1 negative charge SPLL can form tertiary complexes with binary DNA/PLL complexes and make the complexes negative. Meanwhile adding PEG-g-PLG into DNA/PLL complex resulted in successfully shielding the $\delta$-potential of complex at the PLG to DNA ratio above 1 (Figure 3A). If adding excessive PLL into the complex, ie, the total positive charge to negative charge ratio is more than 1 , it was shown that the $\delta$-potential of complex containing $\mathrm{PEG}_{20000}$-g-PLG or $\mathrm{PEG}_{10000}$-g-PLG obviously was lower 

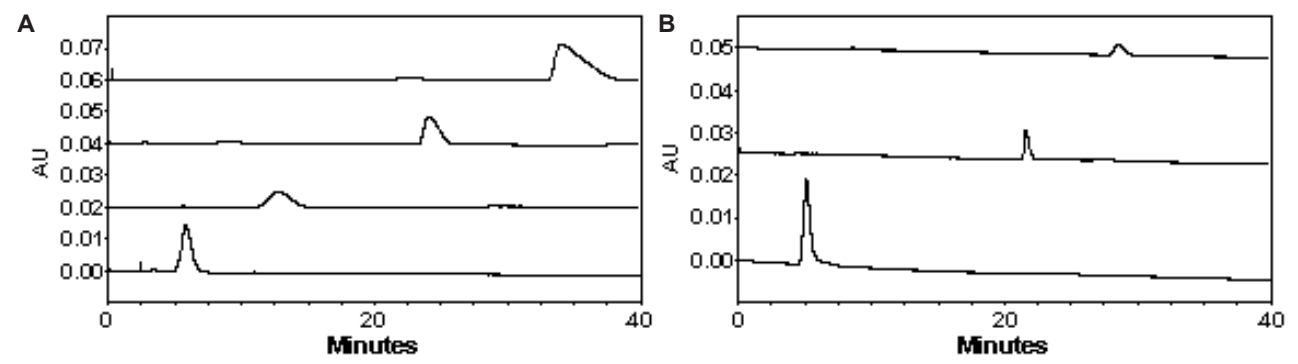

Figure I (A)The electropherogram of PLG (a), PEG ${ }_{5000}-\mathrm{g}-\mathrm{PLG}$ (b), $\mathrm{PEG}_{10000^{-}} \mathrm{g}-\mathrm{PLG}$ (c), $\mathrm{PEG}_{20000}$-g-PLG (d). Running conditions: UV detection (the wavelength of 2 I 4 nm); coated capillary with a effective length of $50 \mathrm{~cm}, 75 \mu \mathrm{m}$ I.D; phosphated buffer of $30 \mathrm{mM}$, pH 8.5; injected at 0.5 psi for 5 sec; separated at - I0 kv. (B) The electropherogram of PLG (a), EGF-g-PLG (b), EGF (c). Running conditions: UV detection (the wavelength of $214 \mathrm{~nm}$ ); coated capillary with a effective length of $50 \mathrm{~cm}, 75 \mu \mathrm{m}$ I.D; phosphated buffer of $40 \mathrm{mM}, \mathrm{pH} 8.0$; injected at $0.5 \mathrm{psi}$ for 5 sec; separated at $-10 \mathrm{kv}$.

than that containing $\mathrm{PEG}_{5000}$-g-PLG. Below this ratio, the $\delta$-potential of complex formed with different molecule weight of PEG-g-PLG didn't differ from each other (Figure 3B).

\section{Nuclease resistance of DNA complexes}

One concern of this system was that polyanion may dissociate DNA complexes by competing with DNA for binding to the polycation (Trubetskoy et al 1999). Another concern was whether PLG or PEG-g-PLG could substitute DNA from complexes or not. It has been shown that DNA could be released from the complexes by adding certain amount of polyanion to the complexes (Katayose and Kataoka 1998;
Koyama et al 2002). Here it was shown that complexes containing $\mathrm{PEG}_{20000}$-g-PLG or $\mathrm{PEG}_{10000}$-g-PLG had stronger protection ability, in which DNA was not or a little digested by DNase I, than complexes containing PEG $_{5000}$-g-PLG at positive to negative charge ratio of 1 (Figure 4). This was probably due to the formation of PEG palisade surrounding the ion-complex core of DNA and PLL segment of the block copolymer (Katayose and Kataoka 1998).

\section{Cell transfection assay}

At total positive to negative charge ratio below 1 , the transfection efficiency of complex was similar to control
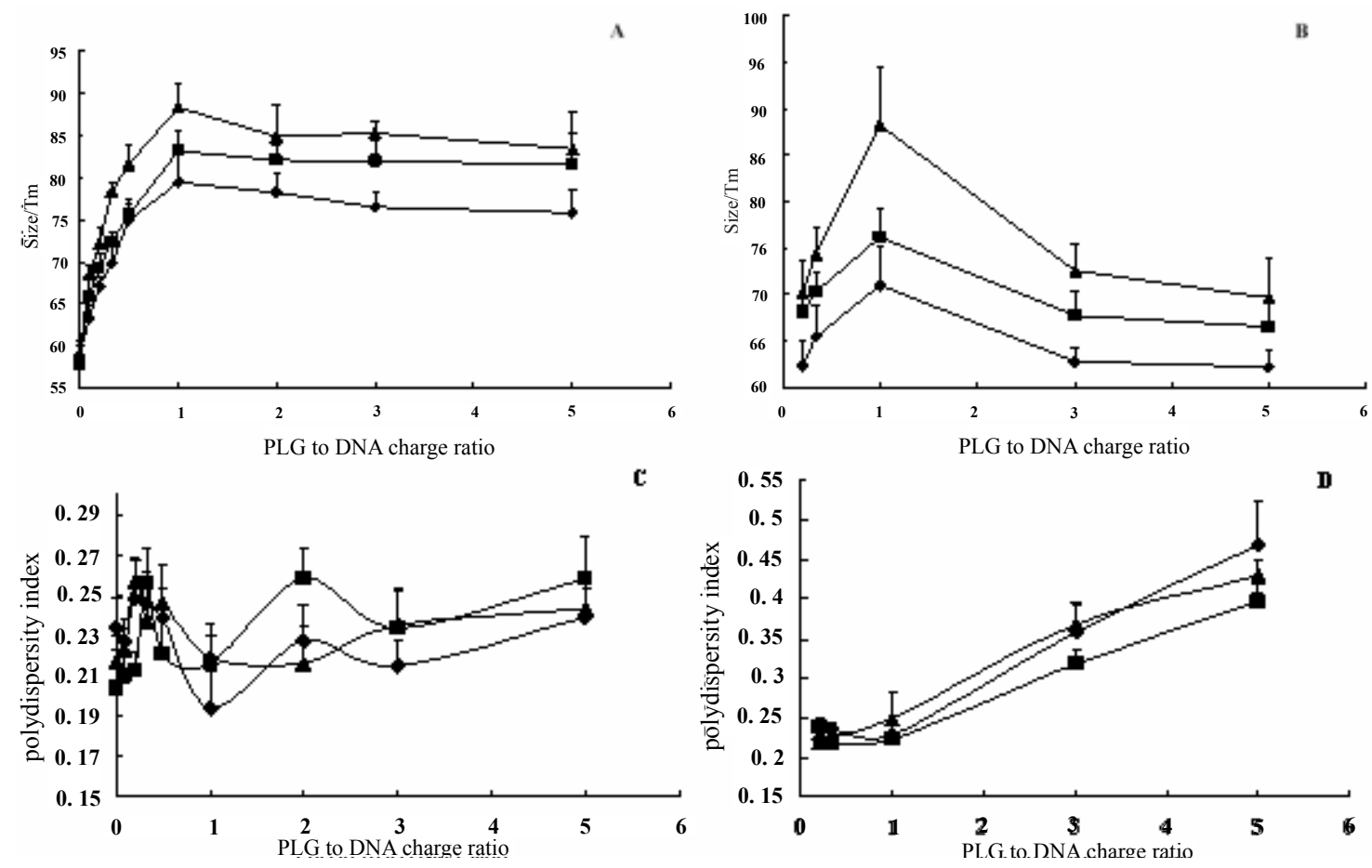

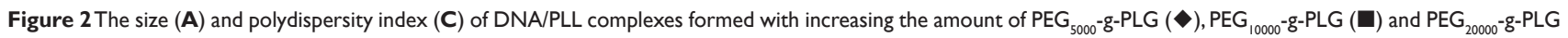
$(\boldsymbol{\Delta})$ at N/P ratio of 2 res pectively. The size $(\mathbf{B})$ and polydispersity index (D) of DNA/PLL complexes formed with $P E G_{5000}{ }^{-g}-P L G(\diamond), P E G{ }_{10000}{ }^{-g}-P L G(\square)$ and PEG ${ }_{20000^{-g}-P L G}$ (A) at total positive to negative charge ratio of I respectively. 

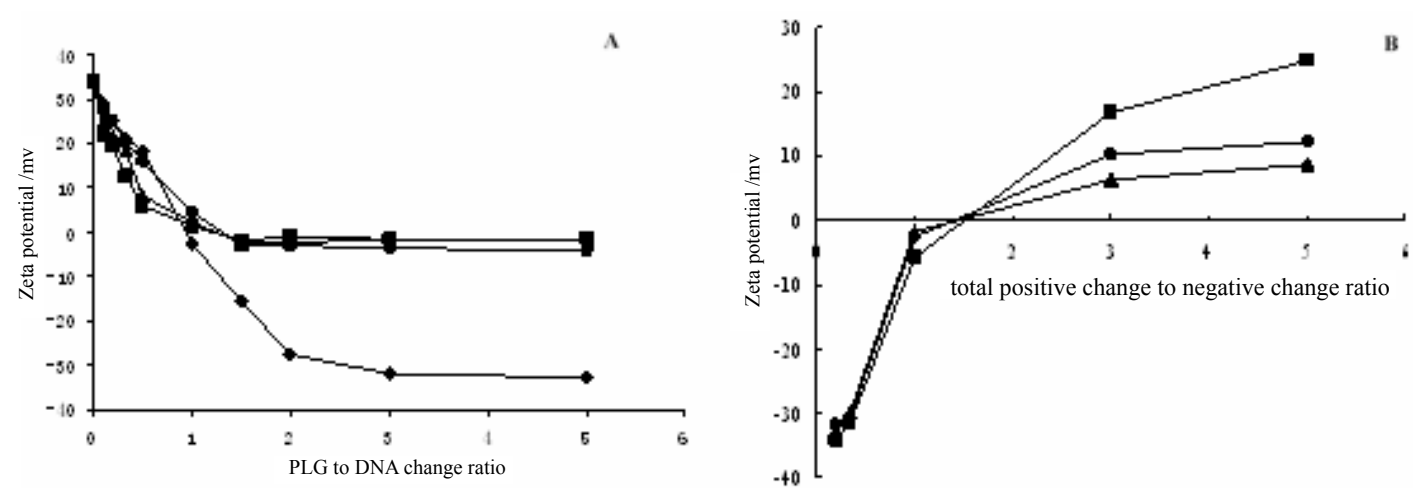

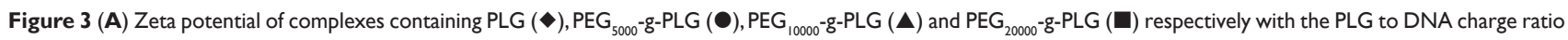

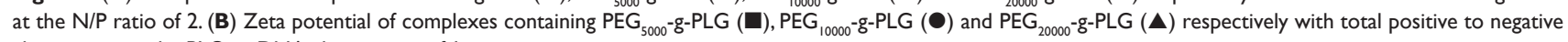
charge ratio at the PLG to DNA charge ratio of $I$.

(Figure 5A), because when the charge amount of PLL was fewer than that of DNA, $\mathrm{PEG}_{20000}$-g-PLG and EGF hardly attached to the surface of complexes with negative charge. Meanwhile at total positive to negative charge ratio of 1 , the complex showed the maximum transfection efficiency which was about 10 times that of control. In comparison, at total positive to negative charge ratio of 3 and 5 , the transfection efficiency gradually decreased. This was due to that when the charge amount of PLL was more than that of DNA, $\mathrm{PEG}_{20000}$-g-PLG and EGF-g-PLG could adsorb to both DNA/PLL complexes and excess PLL. This is the similar to the transfection results with different charge ratio

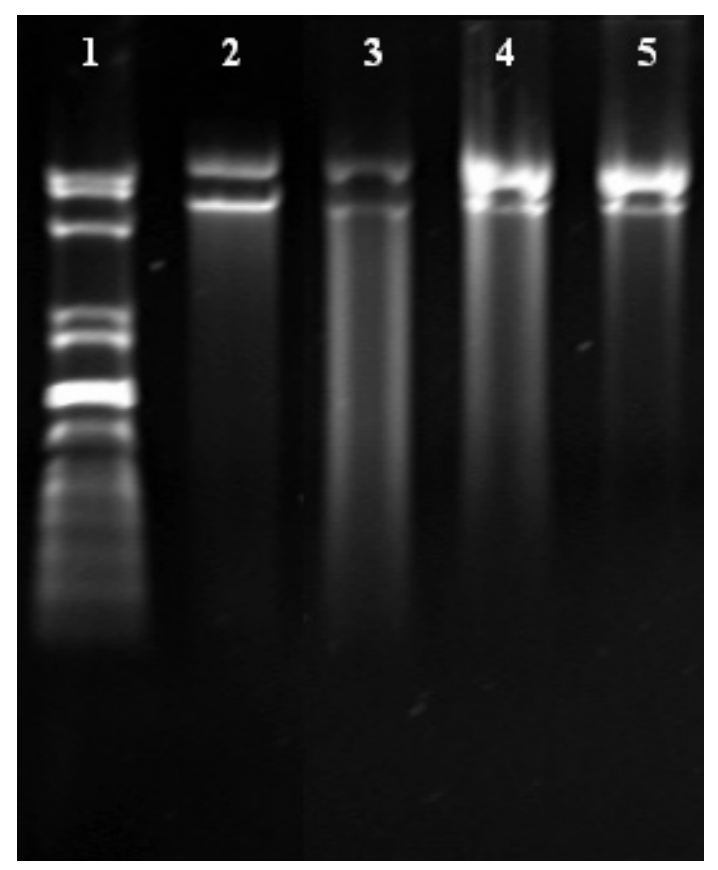

Figure 4 The effect of different molecular PEG-g-PLG on the resistance of complex to the digestion of DNase I at N/P ratio of 2 and the PLG to DNA charge ratio of I. I. marker, 2. DNA, 3. PEG ${ }_{5000}-\mathrm{g}-\mathrm{PLG}, 4$. PEG 10000 -g-PLG and 5. PEG ${ }_{20000}$-g-PLG. of DNA to PLG at total positive to negative charge ratio of 1 (Figure 6B). Surplus PLL could competitively bind with $\mathrm{PEG}_{20000}$-g-PLG and EGF-g-PLG, because PLG was added into DNA/PLL complexes after DNA and PLL was mixed to form complexes, although the charge amount of PLL was equal to that of DNA and PLG.

The transfection efficiency of complexes containing $\mathrm{PEG}_{5000}$-g-PLG was lower than complexes containing other length of PEG-g-PLG at the presence or absence of serum (Figure 5B), in which the transfection efficiency of complexes containing $\mathrm{PEG}_{10000}$-g-PLG or $\mathrm{PEG}_{20000}$-g-PLG was about 20 to 25 times higher than control, probably because enzyme in cell could digest DNA without or with little protection. This was consistent with the result obtained from the assay of DNase I digestion in which complexes containing $\mathrm{PEG}_{5000}$-g-PLG have the worst resistance to DNase I among these complexes (Figure 4). Only EGF on the surface of complexes could obviously increase the transfection efficiency of complexes which was higher than both $\mathrm{PEG}_{20000}$-g-PLG and EGF-g-PLG added into complexes in the medium without serum (Figure 6A). This was probably because the binding of EGF with EGFR on cell was influenced by the steric effect of $\mathrm{PEG}_{20000}$-g-PLG. But at medium with serum $\mathrm{PEG}_{20000}$-g-PLG could protect complexes from attacking of protein in serum, so the transfection efficiency of complexes containing $\mathrm{PEG}_{20000}$-g-PLG and EGF-g-PLG was higher than complexes only containing EGF-g-PLG at this condition.

\section{Conclusions}

In summary, we presented a novel modification method for gene delivery system in which PLG was used as surface modification loading backbone, to which PEG or EGF was linked, to form complexes with PLL and 

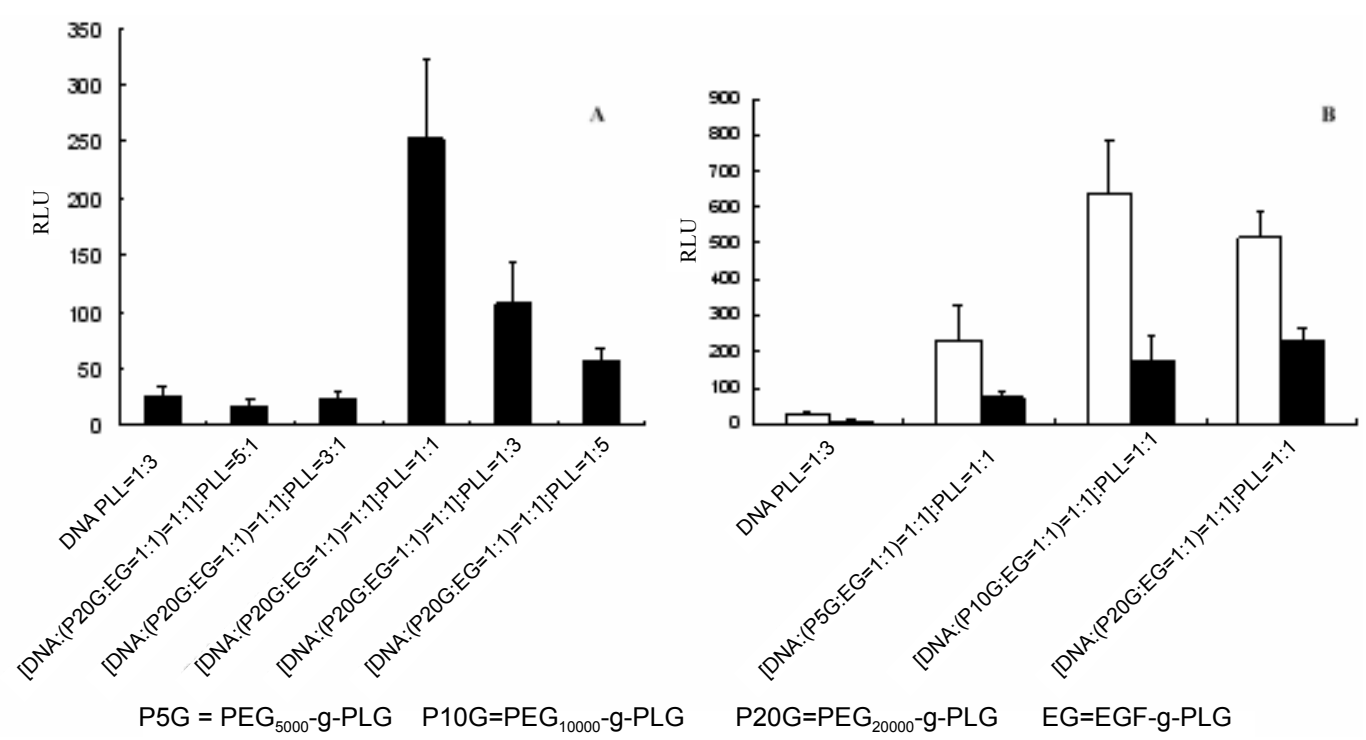

Figure 5 (A) The transfection efficiency of complexes containing PEG ${ }_{2000}$-g-PLG and EGF-g-PLG with total positive to negative charge ratio at the presence of serum. (B) The transfection efficiency of complexes containing PLG conjugated different length of PEG at the presence ( $\square$ ) or absence ( $\square$ ) of serum.

DNA. This system possessed an appreciable advantage of modulating the amount of PEG and/or targeting peptide on the surface of complexes without the need to synthesize a new copolymer containing PEG and/or targeting peptide for each formulation. By using this method, it should be possible to determine the correlation between biological property or physicochemical property of complexes and the amount of PEG and/or targeting peptide in complexes and determine the best complex with optimal transfection efficiency. Although this system provided certain enhancement in cell transfection efficiency, some modification on this system should be taken. For example excessive PLL in DNA/PLL complexes had better be removed to decrease the effect of excessive PLL on the adsorption of PLG conjugates to the surface of DNA/PLL complexes.

\section{Acknowledgments}

The genuine acknowledgement was given to Prof. Yuhong Xu for her support in research and providing experiment apparatus in this studying. Also the authors thank Dr Jinliang Peng
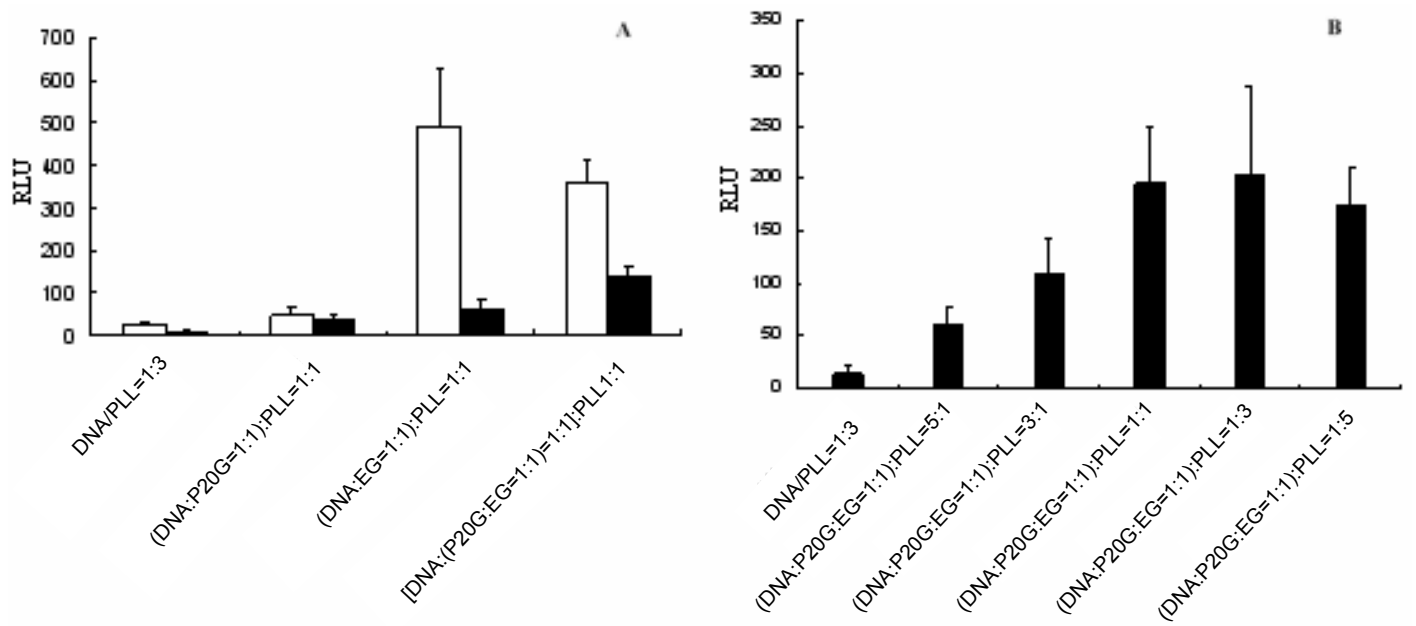

${ }_{\mathrm{P} 20 \mathrm{G}}=\overline{\mathrm{PEG}}_{2000}-\mathrm{g}-\mathrm{PLG} \quad \overline{\mathrm{EG}}=\overline{\mathrm{EG}} \overline{\mathrm{PF}}-\mathrm{g}-\overline{\mathrm{PLG}} \overline{\mathrm{P}}$

Figure $6(\mathbf{A})$ The transfection of complexes formed with different component at the presence $(\square)$ or absence ( $\square$ ) of serum. (B) The transfection efficiency of complexes with different amount of $\mathrm{PEG}_{20000}$-g-PLG and EGF-g-PLG at N/P ratio of 2 and the presence of serum. 
for generous gift of plasmid DNA, Dr Zhonghai Li and Mrs. Ruijiao Zhao at Shanghai Cancer Institute for their direction in cell culture and a gift of human hepatoma cell line SMMC7721. The author report no conflicts of interest in this study.

\section{References}

Finsinger D, Remy JS, Erbacher P, et al. 2000. Protective copolymers for nonviral gene vectors: synthesis, vector characterization and application in gene delivery. Gene Ther, 7:1183-92.

Guo Y, Sun Y, Li G, et al. 2004. The molecular structures of poly(ethylene glycol)-modified nonviral gene delivery polyplexes. Mol Pharm, 1:477-82.

Katayose S, Kataoka K. 1998. Remarkable increase in nuclease resistance of plasmid DNA through supramolecular assembly with poly(ethylene glycol)-poly(L-lysine) block copolymer. J Pharm Sci, 87:160-3.

Koyama Y, Ito T, Matsumoto H, et al. 2003. Novel poly(ethylene glycol) derivatives with carboxylic acid pendant groups: synthesis and their protection and enhancing effect on non-viral gene transfection systems. J Biomater Sci Polymer Edn, 14:515-31.

Koyama Y, Yamada E, Ito T, et al. 2002. Sugar-containing polyanions as a self-assembled coating of plasmid/polycation complexes for receptormediated gene delivery. Macromol Biosci, 2:251-6.

Lee M, Han S, Ko KS, et al. 2001. Repression of GAD autoantigen expression in pancreas beta-Cells by delivery of antisense plasmid/PEG-g-PLL complex. Mol Ther, 4:339-346.
Maruyama K, Iwasaki F, Takizawa T, et al. 2004. Novel receptor-mediated gene delivery system comprising plasmid/protamine/sugar-containing polyanion ternary complex. Biomaterials, 25:3267-73.

Merdan T, Kopecek J, Kissel T. 2002. Prospects for cationic polymers in gene and oligonucleotide therapy against cancer. Adv Drug Deliv Rev, $54: 715-58$

Ogris M, Wagner E. 2002. Targeting tumors with non-viral gene delivery systems. Drug Discov Today, 15:479-85.

Sukhorukov GB, Donath E, Davis S, et al. 1998. Stepwise polyelectrolyte assembly on particle surfaces: a novel approach to colloid design. Polym Adv Technol, 9:759-67.

Trubetskoy VS, Loomis A, Hagstrom JE, et al. 1999. Layer-by-layer deposition of oppositely charged polyelectrolytes on the surface of condensed DNA particles. Nucleic Acids Res, 27:3090-5.

Trubetskoy VS, Wong SC, Subbotin V, et al. 2003. Recharging cationic DNA complexes with highly charged polyanions for in vitro and in vivo gene delivery. Gene Ther, 10:261-71.

Wagner E, Zenke M, Cotten M, et al. 1990. Transferrin-polycation conjugates as carriers for DNA uptake into cells. Proc Nati Acad Sci USA, 87:3410-14.

Zaitsev S, Cartier R, Vyborov O, et al. 2004. Polyelectrolyte nanoparticles mediate vascular gene delivery. Pharm Res, 21:1656-61.

Zhang P, Ren J, Shen Z. 2004. A new quantitative method for circulating DNA level in human serum by capillary zone electrophoresis with laserinduced fluorescence detection. Electrophoresis, 25:1823-8. 